\title{
Signable Posets and Partitionable Simplicial Complexes*
}

\author{
P. Kleinschmidt ${ }^{1}$ and S. Onn ${ }^{2}$ \\ ${ }^{1}$ Universität Passau, 94030 Passau, Germany \\ kleinsch@winf.uni-passau.de \\ ${ }^{2}$ Technion-Israel Institute of Technology, 32000 Haifa, Israel \\ onn@ie.technion.ac.il
}

\begin{abstract}
The notion of a partitionable simplicial complex is extended to that a signable partially ordered set. It is shown in a unified way that face lattices of shellable polytopal complexes, polyhedral cone fans, and oriented matroid polytopes, are all signable. Each of these classes, which are believed to be mutually incomparable, strictly contains the class of convex polytopes. A general sufficient condition, termed total signability, for a simplicial complex to satisfy McMullen's Upper Bound Theorem on the numbers of faces, is provided. The simplicial members of each of the three classes above are concluded to be partitionable and to satisfy the upper bound theorem. The computational complexity of face enumeration and of deciding partitionability is discussed. It is shown that under a suitable presentation, the face numbers of a signable simplicial complex can be efficiently computed. In particular, the face numbers of simplicial fans can be computed in polynomial time, extending the analogous statement for convex polytopes.
\end{abstract}

\section{Introduction}

The extensively studied notion of shellability is of basic importance for the combinatorial, enumerative, and algorithmic study of simplicial complexes and posets [1], [2], [4], [6], [16]. However, there are important classes of complexes which are not shellable, but do possess the somewhat weaker property of partitionability. This property often suffices to lead to efficient computation of the face numbers of such complexes, and to derive properties of these numbers and inequalities satisfied by them. In particular, the $h$ numbers of a partitionable complex, important numerical invariants which determine its face numbers, admit a combinatorial interpretation and are nonnegative.

\footnotetext{
* The research of S. Onn was supported by the Alexander von Humboldt Stiftung, by the Fund for the Promotion of Research at the Technion, and by Technion VPR Fund 191-198.
} 
In this article we take on a systematic study of partitionable simplicial complexes and their face lattices. We extend our study to face lattices of nonsimplicial complexes and more generally to partially ordered sets, and introduce the notion of a signable poset, which naturally extends the notion of partitionability. We show in a unified way that important classes of posets are signable, which in particular implies that the simplicial members of these classes are partitionable:

- Theorem 3.6. Shellable posets (hence shellable polytopal complexes) are signable.

- Theorem 4.5. Polyhedral fans are signable.

- Theorem 5.11. Oriented matroid polytopes are signable.

We provide a general, purely combinatorial, sufficient condition for a simplicial complex to satisfy McMullen's Upper Bound Theorem on the number of faces. This condition holds for the simplicial members of all three classes above. We thank a referee for the present more elegant formulation of this result:

- Theorem 4.4. Any totally signable simplicial complex satisfies the Upper Bound Theorem.

Finally, we show that under a suitable presentation, the face numbers of a signable simplicial complex can be efficiently computed (Lemma 6.2). This applies in particular to simplicial fans presented by their maximal cones, extending the analogous statement for convex polytopes:

- Theorem 6.4. The face numbers of simplicial polyhedral fans are polynomial time computable.

Each of the main classes of complexes and posets under study (see Fig. 1), namely shellable polytopal complexes, polyhedral cone fans (also known as spherical polytopes), and oriented matroid polytopes, strictly contains the class of convex polytopes: every polytope $K$ is line-shellable; it induces the oriented matroid polytope of affine dependencies on its vertices; and it induces the normal-fan of its polar $K^{*}$, namely the fan of cones polar to faces of $K^{*}$. These three classes are believed to be mutually incomparable (see discussion in the last section). In particular, the problem of showing that fans are not shellable in general is outstanding; some progress on that was recently achieved by Mani [12], who reduced the problem to a certain conjecture in differential geometry. Likewise, it is an open question whether oriented matroid polytopes are shellable [3], and the answer is again likely to be negative (surprisingly, the face lattice of an oriented matroid polytope turned upside down is shellable - this is a result of Edmonds and Mandel-see [3]). In contrast we show that all three classes are signable. This shows in particular that simplicial oriented matroid polytopes are partitionable and satisfy McMullen's Upper Bound Theorem, and generalizes the results of [9] on simplicial fans. We also discuss the computational complexity of deciding shellability and partitionability, and the complexity of face enumeration. In a continuation article [15], poset signings are further investigated, and are shown to play an important role in the algorithmic and enumerative study of recursively signable posets, their flag vectors, and their chain complexes and barycentric subdivisions.

Our article is organized as follows. The next section provides a brief review of simplicial complexes, their face numbers, shellability, and partitionability. In Section 3 we 
define the notion of a signable poset, which naturally extends that of a partitionable simplicial complex. We show that any shellable poset (one admitting a recursive coatom ordering) is signable and conclude (Corollary 3.7), based on results in Björner-Wachs [4], that shellable polytopal complexes are signable.

In Section 4 we set up the framework of a signed family, which provides a systematic way of proving poset signability. We illustrate it on (nonoriented) matroid complexes (Example 4.3), and use it to prove that polyhedral fans are signable (Theorem 4.5), which stands in contrast with the common belief that they are not shellable in general. Also in Section 4, we show that if a signable simplicial complex is moreover totally signable, then it satisfies McMullen's Upper Bound Theorem on the face numbers [13]. Thus, Theorem 4.4 provides a rather general sufficient condition for a simplicial complex to satisfy this theorem.

In Section 5 we use the framework of signed families once more to prove that (Las Vergnas face lattices of) oriented matroid polytopes are signable (Theorem 5.11), again in contrast with the common belief that they are not shellable in general. We then apply Theorem 4.4 to conclude that simplicial oriented matroid polytopes satisfy the Upper Bound Theorem (Theorem 5.12).

In Section 6 the computational complexity of face enumeration is discussed, as well as the complexity of deciding shellability and partitionability. In contrast with the $\# \mathcal{P}$ completeness of the face numbers of a polytope [11], for which we give a short new proof (Proposition 6.1), we have Lemma 6.2; it asserts that there is a polynomial-time algorithm that, for a suitably presented signable simplicial complex, computes the face numbers. This is used to show (Theorem 6.4) that the face numbers of simplicial fans can be computed in polynomial time, extending the analogue for simplicial polytopes.

We finish in Section 7 with some remarks and open questions, in particular regarding the hierarchy of various classes of complexes and posets considered in this article and depicted in Fig. 1.

\section{Some Preliminaries}

Here we briefly review some terminology on simplicial complexes and their $f$-vectors and $h$-vectors, and the notions of shellability and partitionability of a simplicial complex. By a simplicial complex $\Delta$ throughout we mean a finite pure-dimensional one. The dimension of a face $F \in \Delta$ is taken as the number of vertices in it (one unit more than the geometrical dimension). The faces of maximal dimension are the facets of $\Delta$, and the faces of codimension 1 are the ridges of $\Delta$.

A partial shelling of $\Delta$ is a sequence $F_{1}, \ldots, F_{k}$ of some of the facets of $\Delta$ such that for all $1 \leq i<j \leq k$ there is $r<j$ such that $F_{i} \cap F_{j} \subseteq F_{r} \cap F_{j}$ and $F_{r} \cap F_{j}$ is a ridge of $\Delta$. A shelling of $\Delta$ is a partial shelling consisting of all its facets, and $\Delta$ is shellable if it admits a shelling.

A facing of $\Delta$ is an assignment $\varphi(F) \subseteq F$ of a face to each facet $F$ in $\Delta$. A facing of $\Delta$ is exact if it induces a partitioning of $\Delta$ in the following way: for any face $G \in \Delta$ there is a unique facet $F$ in $\Delta$ for which $\varphi(F) \subseteq G \subseteq F$ holds. A simplicial complex $\Delta$ is partitionable if it admits an exact facing. If $F_{1}, \ldots, F_{n}$ is a shelling of $\Delta$, then, letting $\varphi\left(F_{i}\right)$ be the intersection of all ridges of $F_{i}$ not contained in any $F_{j}(j<i)$, it is easily 
verified that $\varphi$ is an exact facing of $\Delta$. Thus, shellability implies partitionability; but the converse is false, as is demonstrated by the following example.

Example 2.1 (The Real Projective Plane). Following is the list of facets of a nonshellable triangulation $\Delta$ of the real projective plane with six vertices; an exact facing of $\Delta$ is indicated by overlining, in each facet $F$, the vertices constituting $\varphi(F)$ :

\section{$\begin{array}{llllllllll}123 & 12 \overline{6} & 13 \overline{5} & \overline{145} & 1 \overline{46} & 23 \overline{4} & 2 \overline{45} & \overline{25} 6 & \overline{3} 4 \overline{6} & 3 \overline{56} .\end{array}$}

If $\Delta$ is $d$-dimensional, then its $f$-vector is the face enumerator $f=\left(f_{0}, \ldots, f_{d}\right)$, where $f_{i}$ is the number of $i$-faces of $\Delta$. Its $h$-vector is the vector $h=\left(h_{0}, \ldots, h_{d}\right)$ related to the $f$-vector via the invertible pair of linear transformations given by

$$
f_{i}=\sum_{j=0}^{i}\left(\begin{array}{l}
d-j \\
d-i
\end{array}\right) h_{j} \quad \text { and } \quad h_{i}=\sum_{j=0}^{i}(-1)^{i-j}\left(\begin{array}{c}
d-j \\
d-i
\end{array}\right) f_{j}
$$

In many situations the $h$-vector is more suitable for the enumerative study of a simplicial complex than the $f$-vector itself. For example, McMullen's upper-bound theorem [13] asserts, in terms of the $h$-vector, that for a simplicial polytope

$$
h_{i} \leq\left(\begin{array}{c}
h_{1}+i-1 \\
i
\end{array}\right)
$$

holds for all positive $i$. A simplicial complex for which these inequalities hold is said to possess the upper-bound property.

In the case of a partitionable complex, the $h$-vector has the following well-known direct combinatorial interpretation.

Proposition 2.2. If $\varphi$ is an exact facing of a d-dimensional simplicial complex $\Delta$, then its $h$-vector is given by

$$
h_{j}=\mid\{F \in \Delta: F \text { facet }, \operatorname{dim} \varphi(F)=j\} \mid, \quad j=0, \ldots, d .
$$

Proof. Let $\varphi$ be an exact facing of $\Delta$. If $F$ is a facet of $\Delta$ with $\operatorname{dim} \varphi(F)=j$, then the number of $i$-faces $G$ satisfying $\varphi(F) \subseteq G \subseteq F$ is zero if $i<j$ and is $\left(\begin{array}{c}d-j \\ i-j\end{array}\right)=\left(\begin{array}{c}d-j \\ d-i\end{array}\right)$ otherwise. Thus,

$$
f_{i}=\sum_{j=0}^{i}\left(\begin{array}{l}
d-j \\
d-i
\end{array}\right) \mid\{F \in \Delta: F \text { facet, } \operatorname{dim} \varphi(F)=j\} \mid
$$

The claim follows by comparing this with the invertible expression of the $f$-vector in terms of the $h$-vector given above.

Thus, in Example 2.1, the $h$-vector is read off from the indicated facing to be $h=$ $(1,3,6,0)$, from which the $f$-vector is easily computed to be $f=(1,6,15,10)$. 


\section{Signable and Shellable Posets}

In this section we define the notion of a signable partially ordered set (poset), which generalizes the notion of a partitionable simplicial complex. We also show that any shellable poset-one admitting a recursive coatom ordering [4]-is signable. In the following sections we establish the signability of various other important classes of posets.

We start by defining the signability of a simplicial complex. A signing of a simplicial complex $\Delta$ is an assignment $\chi(R, F) \in\{-,+\}$ of a sign to each pair of a ridge $R$ and a facet $F$ containing it. A ridge $R$ is positive in a facet $F$ containing it if $\chi(R, F)=+$, and is negative in $F$ otherwise. Also, a vertex $v$ is positive in a facet $F$ containing it if $\chi(F-v, F)=+$, and is negative in $F$ otherwise. A facet is positive if all its ridges are positive in it. Recall that the link of a face $G$ in $\Delta$ is the simplicial complex

$$
\mathrm{lk}_{\Delta}(G)=\{H-G: G \subseteq H \in \Delta\} .
$$

The complex itself is the link $1 \mathrm{k}_{\Delta}(\emptyset)$ of its empty face. The link $\mathrm{lk}_{\Delta}(G)$ of any face $G \in \Delta$ naturally inherits a signing from the signing $\chi$ of $\Delta$, given by $\chi(R-G, F-G)=$ $\chi(R, F)$ for all ridge-facet pairs in $\mathrm{lk}_{\Delta}(G)$. Thus, $F-G$ is a positive facet of $\mathrm{lk}_{\Delta}(G)$ under $\chi$ if, for all ridges $R$ satisfying $G \subseteq R \subset F, \chi(R-G, F-G)=\chi(R, F)=+$ holds. A signing $\chi$ of $\Delta$ is exact if the link of any face $G \in \Delta$ contains a unique facet which is positive under $\chi$. A simplicial complex is signable if it admits an exact signing. There is an obvious correspondence between signings and facings of $\Delta$, where the facing $\varphi$ corresponding to a signing $\chi$ is given by

$$
\varphi(F)=\bigcap\{R \subset F: R \text { ridge, } \chi(R, F)=+\} .
$$

Equivalently, $\varphi(F)$ consists of the negative vertices of $F$.

Proposition 3.1. A simplicial complex is partitionable if and only if it is signable.

Proof. Let $\chi$ be any signing of $\Delta$, and let $\varphi$ be the corresponding facing of $\Delta$ as above. We claim that, for any face $G \in \Delta$, a facet $F-G$ of $\operatorname{lk}_{\Delta}(G)$ is positive in $\mathrm{lk}_{\Delta}(G)$ under $\chi$ if and only if $\varphi(F) \subseteq G \subseteq F$ holds for the facet $F$ of $\Delta$. Indeed, the latter is equivalent to the condition

$$
\begin{aligned}
\varphi(F) & =\bigcap\{R \subset F: R \text { ridge, } \chi(R, F)=+\} \\
& \subseteq \bigcap\{R \subset F: R \text { ridge, } G \subseteq R\}=G \subseteq F,
\end{aligned}
$$

which holds if and only if all ridges $R \subset F$ containing $G$ are positive in $F$, i.e., if $F-G$ is positive in $\mathrm{lk}_{\Delta}(G)$ under $\chi$. Thus, $F-G$ is the unique positive facet in $1 \mathrm{k}_{\Delta}(G)$ under the signing $\chi$ if and only if $F$ is the unique facet of $\Delta$ satisfying $\varphi(F) \subseteq G \subseteq F$. Hence $\chi$ is exact if and only if $\varphi$ is.

Note that, in the notation of a partitioning as in Example 2.1, the signing $\chi$ of $\Delta$ is directly read off: a vertex $v$ (and the ridge $R-v$ ) is negative in a facet $F$ if it is overlined in $F$. Also, Proposition 2.2 can be reformulated as follows. 
Proposition 3.2. If $\chi$ is an exact signing of $\Delta$, then its $h$-vector is given by

$$
h_{k}=\mid\{F \in \Delta: F \text { is a facet containing } k \text { negative ridges }\} \mid \text {. }
$$

This immediately gives the following consequence regarding the so-called Dehn-Sommerville relations.

Proposition 3.3. If a signable d-dimensional simplicial complex $\Delta$ has an exact signing $\chi$ whose negative $-\chi$ is also exact, then $\Delta$ satisfies the Dehn-Sommerville relations $h_{k}=h_{d-k}$ for $k=0, \ldots, d$.

We now generalize the above notions to partially ordered sets. The terminology on partially ordered sets is mostly drawn from [18]. Throughout this article, by a poset we mean a finite graded partially ordered set $P$ with a unique minimal element 0 and a unique maximal element 1 . The rank function of $P$ will be denoted by $r$, so that $r(0)=0$ and $r(P)=r(1)$, which will usually be assumed to be $d+1$. The order relation in $P$ will be denoted by $x \leq y$ (or $x<y$ if $x \neq y$ ), whereas the covering relation will be dentoed by $x \prec y$. The atoms of $P$ are the covers of 0 , i.e., elements of rank 1 , and the coatoms of $P$ are the cocovers of 1, i.e., elements of corank 1 . The cocoatoms are the cocovers of coatoms, i.e., elements of corank 2 . The closed interval corresponding to elements $x \leq y$ in $P$ is $[x, y]=\{z \in P: x \leq z \leq y\}$, which is itself a poset of rank $r[x, y]=r(y)-r(x)$.

A signing of a poset $P$ is an assignment $\chi(x, y) \in\{-,+\}$ of a sign to each pair of elements $x, y \in P$ satisfying $x \prec y \prec 1$, i.e., a cocoatom $x$ and a coatom $y$ covering it. A coatom $y$ is positive under $\chi$ if $\chi(x, y)=+$ for all its cocovers $x \prec y$ (if $P$ consists of 0 and 1 only, then 0 is its unique positive coatom). Any upper interval $I=[z, 1]$ of $P$ inherits a signing from $\chi$ by restriction. Since no confusion can occur, we use a signing $\chi$ of a poset $P$ to denote also the inherited signing of any upper interval of $P$. Thus, a coatom $y \in[z, 1]$ is positive in $[z, 1]$ under $\chi$ if $\chi(x, y)=+$ for all $z \leq x \prec y$.

Definition 3.4. A signing $\chi$ is exact if for every $z<1$ in $P$ there is a unique positive coatom $y$ under $\chi$ in the interval $[z, 1]$. A poset is signable if it admits an exact signing.

Note that any poset of rank $r(P) \leq 2$ is signable.

By a simplicial poset we mean the face lattice $P=P(\Delta)$ of a simplicial complex $\Delta$, i.e., the poset of faces of $\Delta$ ordered by inclusion, augmented with a maximal element 1 . The simplicial complex $\Delta$ can be recovered from $P(\Delta)$ by letting its vertex set be the set of atoms of $P$, and, for each $y<1$ in $P$, taking the corresponding face of $\Delta$ to be the set of all atoms $x$ satisfying $x \leq y$. In particular, the coatoms of $P$ correspond to facets of $\Delta$, and the cocoatoms of $P$ correspond to ridges of $\Delta$. Thus, a signing of $\Delta$ as defined earlier can be identified with a signing of its face lattice $P$ in the obvious way. In particular, $P(\Delta)$ is signable if and only if $\Delta$ is partitionable.

Remark. The following partial analogue of Proposition 3.1 holds for posets: if $\chi$ is an exact signing of a poset $P$, and each coatom $y$ of $P$ is assigned the following union of 
upper intervals of $P$ :

$$
\begin{aligned}
\varphi(y) & =\{z \leq y: y \text { is a positive coatom in }[z, 1]\} \\
& =\{z \leq y: \chi(x, y)=+ \text { for all } x \text { such that } z \leq x \prec y\},
\end{aligned}
$$

then the $\varphi(y)$ form a partition of $P \backslash\{1\}$. Details are left to the reader.

We now show that any shellable poset is signable; by a shellable poset we mean one which admits a recursive coatom ordering, e.g., the face lattice of a shellable simplicial complex as defined earlier. Recall the following definition from [4]:

Definition 3.5. A recursive coatom ordering of a poset $P$ is a total ordering of its coatoms such that the following hold:

(1) If $z<y, y^{\prime} \prec 1$ in $P$ and $y$ precedes $y^{\prime}$ in the ordering, then an element $v \in P$ and a coatom $y^{\prime \prime}$ which also precedes $y^{\prime}$ exist such that $z \leq v \prec y^{\prime \prime}, y^{\prime}$.

(2) For every coatom $y$ in $P$, there is a recursive coatom ordering of the interval $[0, y]$ in which the coatoms of $[0, y]$ that come first are those that are covered by some $y^{\prime}$ preceding $y$.

Any total ordering of the coatoms of a poset $P$ naturally induces a signing of $P$ in the following way: for $x \prec y \prec 1$ put $\chi(x, y)=+$ if $y$ is the first coatom covering $x$, and put $\chi(x, y)=-$ otherwise.

Theorem 3.6. The signing induced by a recursive coatom ordering of a poset $P$ is exact. Thus, shellable posets are signable.

Proof. Let $\chi$ be a signing of $P$ induced from a recursive coatom ordering. Let $z$ be any element of $P$, which we may assume to be of corank at least 2. Let $y$ be the first (under the coatom ordering) coatom in the interval $[z, 1]$. If $y$ is not positive in $[z, 1]$, then it has a cocover $x$ in $[z, 1]$ satisfying $\chi(x, y)=-$. However, then the first coatom $y^{\prime}$ covering $x$ is different from $y$ since $\chi\left(x, y^{\prime}\right)=+$, and $y^{\prime}$ is in $[z, 1]$ and precedes $y$, contradicting the assumption that $y$ is the first coatom in $[z, 1]$. Thus, $y$ is a positive coatom in $[z, 1]$. Now consider any other coatom $y^{\prime}$ in $[z, 1]$. By Definition 3.5 above, there is a coatom $y^{\prime \prime}$ preceding $y^{\prime}$ and an element $v$ in $[z, 1]$ such that $v \prec y^{\prime \prime}, y^{\prime}$; but this means that $y^{\prime}$ is not the first coatom covering $v$, so that $\chi\left(v, y^{\prime}\right)=-$, hence $y^{\prime}$ is not a positive coatom in $[z, 1]$. Thus, the first coatom in $[z, 1]$ is the unique positive coatom in it, and $\chi$ is an exact signing of $P$.

The converse of Theorem 3.6 is false: for example, the triangulation of the real projective plane in Example 2.1 is partitionable but not shellable, so its face lattice is signable but does not admit a recursive coatom ordering.

Recall that a polytopal complex is a finite collection of polytopes in Euclidean space, which is closed under taking faces and intersections. It was shown by Björner and Wachs [4] that a polytopal complex is shellable if and only if its face lattice is a shellable poset, i.e., admits a recursive coatom ordering (see [4] for more details). We therefore have the following corollary.

Corollary 3.7. Face lattices of shellable polytopal complexes are signable. 


\section{The Upper-Bound Property and Signability of Polyhedral Fans}

In the first part of this section we introduce some tools that enable systematic proofs of signability, and of the upper-bound property in the simplicial case. First, we set up the framework of a signed family, which allows a systematic proof of signability. Second, we show that if a signable simplicial complex has a rich enough supply of exact signings, then it possesses the upper-bound property (Theorem 4.4). We demonstrate these notions on matroid complexes.

In the second part of this section we use these tools to show that face lattices of polyhedral fans are signable. This extends the results of [9] for the simplicial case. The upper-bound property for fans then follows from Theorem 4.4. In the next section we apply the same tools to oriented matroid polytopes.

A signed poset is a pair $(P, \chi)$ consisting of a poset $P$ equipped with a signing $\chi$ of $P$. Since no confusion can occur, we are usually somewhat sloppy about the distinction between a signed poset and its "underlying unsigned poset," and refer to $P$ both as the poset and the signed poset. The signed upper interval of an element $z$ in a signed poset $P$ is the signed poset $[z, 1]$ equipped with the signing of $[z, 1]$ inherited from the signing $\chi$ of $P$. The isomorphism of signed posets is defined in the obvious way, namely it is a signing preserving poset isomorphism.

Definition 4.1. A signed family is a family $\mathcal{P}$ of signed posets which is closed under taking signed upper intervals. A signed family $\mathcal{P}$ is exact if for every $P \in \mathcal{P}$ there is a unique coatom in $P$ which is positive under the signing $\chi$ of $P$.

Note that a signed family may include several copies of the same poset, equipped with different signings.

Proposition 4.2. Let $\mathcal{P}$ be an exact signed family. Then the signing $\chi$ of every $P \in \mathcal{P}$ is exact. In particular, all posets in $\mathcal{P}$ are signable.

Proof. Let $\mathcal{P}$ be an exact signed family and let $P$ be a member of $\mathcal{P}$ with signing $\chi$. Let $z$ be any element of $P$. Then the signed upper interval $[z, 1]$ is in $\mathcal{P}$, so $[z, 1]$ has a unique positive coatom under $\chi$. Thus, by definition, $\chi$ is an exact signing of $P$.

We illustrate the use of signed families on complexes of independent sets in a matroid. See [1] and [2] for more information on such complexes.

Example 4.3 (Matroid Complexes). Given a matroid $M$, its matroid complex is the simplicial complex $\Delta=\Delta(M)$ of independent sets in $M$, and its face lattice is the face lattice $P(M)=P(\Delta)$ of $\Delta$. The facets of $\Delta$ are the bases of $M$; each ridge $R \in \Delta$ spans a hyperplane of $M$ (a flat of $M$ of corank 1), whose complement in $M$ is the unique cocycle $D$ in $M$ which is disjoint from $R$. Moreover, a subset $R+v$ is a facet of $\Delta$ if and only if $v \in D$. If $M$ is equipped with a total order of its elements, then it is an ordered matroid (see [2]). We endow the face lattice $P=P(M)$ of an ordered matroid $M$ with a signing $\chi=\chi(M)$, thus turning it into a signed poset, as follows: for a ridge $R \in P$ 
set $\chi(R, R+v)$ to be positive if $v$ is the minimal (under the total order of $M$ ) element in the unique cocycle $D$ of $M$ disjoint from $R$, and negative otherwise.

Let $\mathcal{P}$ be the family of signed posets of ordered matroids. Consider any $P=P(M) \in$ $\mathcal{P}$, where $M$ is an ordered matroid. Let $G \in P$ be any face (independent set of $M$ ). Let $M / G$ be the contracted matroid, totally ordered by restriction from $M$. Then its signed poset $P(M / G)$ is a member of $P$, and is easily seen to be isomorphic to the signed upper interval of $G$ in $P$. Thus, $\mathcal{P}$ is a signed family. It is not too hard either to show that, for any member $P=P(M) \in \mathcal{P}$, the facet of $P$ (basis of $M$ ) which is smallest in the lexicographic order induced by the total order on the elements of $M$, is the unique positive facet in $P$ under $\chi(M)$. Thus, $\mathcal{P}$ is an exact signed family. It follows from Proposition 4.2 that all matroid complexes are signable.

We next proceed to the upper-bound property in the simplicial case. Call a simplicial complex $\Delta$, and its face lattice $P(\Delta)$, totally signable or totally partitionable, if for every vertex $v \in \Delta$ an exact signing $\chi_{v}$ of $\Delta$ exists such that $v$ is positive in all facets containing it. Equivalently, for every $v$ an exact facing $\varphi_{v}$ exists such that $v \notin \varphi_{v}(F)$ for all facets $F \in \Delta$. The following theorem provides a rather general sufficient condition for a partitionable simplicial complex to possess the upper-bound property. We thank a referee for the present formulation of this theorem which simplifies our original formulation.

Theorem 4.4. If $\Delta$ is a totally signable simplicial complex, then it possesses the upperbound property.

Proof. The proof is an extension of McMullen's proof for simplicial convex polytopes [13]. Let $\Delta$ be a $d$-dimensional totally signable simplicial complex with $n=f_{1}$ vertices. Recall (Proposition 3.1) that any exact signing $\chi$ of $\Delta$ induces an exact facing of $\Delta$ given by

$$
\varphi(F)=\bigcap\{R \subset F: R \text { ridge, } \chi(R, F)=+\},
$$

and (Proposition 2.2) the $h$-vector of $\Delta$ is given by

$$
h_{i}=\mid\{F \in \Delta: F \text { facet, } \operatorname{dim} \varphi(F)=i\} \mid, \quad i=0, \ldots, d .
$$

In particular, the right-hand side is independent of $\chi$ and $\varphi$.

Note that $h_{0}=1$ and $h_{1}=n-d$, so that the upper-bound property which needs to be proved is

$$
h_{k} \leq\left(\begin{array}{c}
n-d+k-1 \\
k
\end{array}\right), \quad k=0, \ldots, d .
$$

For each vertex $v$ of $\Delta$, write $h_{i}(v)$ for $h_{i}\left(\mathrm{lk}_{\Delta}(v)\right)$. We prove the following two relations for $i=0, \ldots, d-1$ :

$$
\begin{aligned}
& h_{i}(v) \leq h_{i} \quad \text { for all vertices } v \text { of } \Delta \\
& \sum_{v \in \text { vert } \Delta} h_{i}(v)=(i+1) h_{i+1}+(d-i) h_{i}
\end{aligned}
$$


The upper-bound property is then proved by applying (1) and (2) successively as follows:

$$
\begin{aligned}
h_{k} \leq & \left(\frac{n-d+k-1}{k}\right) h_{k-1} \leq \frac{(n-d+k-1)(n-d+k-2)}{k(k-1)} h_{k-2} \leq \cdots \\
& \leq \frac{(n-d+k-1)(n-d+k-2)}{k(k-1)} \cdots \frac{(n-d)}{1} h_{0}=\left(\begin{array}{c}
n-d+k-1 \\
k
\end{array}\right), \\
& k=1, \ldots, d .
\end{aligned}
$$

To prove (1) we choose for $v$ an exact signing $\chi$ of $\Delta$ under which $v$ is positive in all facets containing it. Such a signing exists by our assumption and its choice does not change $h_{i}(v)$ and $h_{i}$. Let $F$ be a facet containing $v$. Now the facing $\varphi$ of $\Delta$ is given by

$$
\varphi(F)=\bigcap\{R \subset F: R \text { ridge, } \chi(R, F)=+\},
$$

and the facing $\varphi_{v}$ of $\mathrm{lk}_{\Delta}(v)$ (coming from the signing of $\mathrm{lk}_{\Delta}(v)$ inherited from $\chi$ ) is given by

$$
\varphi(F-v)=\bigcap\{R-v \subset F-v: R \text { ridge containing } v, \chi(R, F)=+\} ;
$$

as $\chi(F-v, F)=+$, we find that $\varphi_{v}(F-v)=\varphi(F)$. It follows that if $F-v$ contributes to $h_{i}(v)$, i.e. $\operatorname{dim} \varphi_{v}(F-v)=i$, then $F$ also contributes to $h_{i}$. This proves (1).

To prove (2), let $\chi$ be any exact signing of $\Delta$. Then a facet which contributes to $h_{i}$ has $d-i$ positive ridges and hence $d-i$ vertices for which it contributes to

$$
\sum_{v \in \text { vert } \Delta} h_{i}(v)
$$

via the facet $F-v$ of $\mathrm{lk}_{\Delta}(v)$; and a facet which contributes to $h_{i+1}$ has $i+1$ negative vertices for which it contributes to

$$
\sum_{v \in \text { vert } \Delta} h_{i}(v)
$$

As there are no other types of facets contributing to the left-hand side of (2), this completes the proof of (2) and the proof of the theorem.

We apply this theorem to the example of matroid complexes.

Example 4.4 (continued). We want to show that matroid complexes are totally signable. Let $M$ be a matroid and let $\Delta=\Delta(M)$ be its matroid complex. Let $v$ be any vertex of $\Delta$ (an element of $M$ ). Choose a total order on the elements of $M$ under which $v$ is the minimal element. Let $\chi_{v}=\chi(M)$ be the exact signing of the face lattice $P=P(M)$ of the ordered matroid $M$ as defined earlier. Then it is easy to see that $v$ is positive under $\chi_{v}$ in all facets of $P$ (bases of $M$ ) which contain it. It follows that all matroid complexes are totally signable, and so, by Theorem 4.4, possess the upper-bound property. 
Next, we turn to establish the signability of polyhedral cone fans. A fan in Euclidean space $\mathbb{R}^{d}$ is a finite collection $\mathcal{S}$ of pointed polyhedral cones which is closed under taking faces and intersections, and which covers $\mathbb{R}^{d}$. The face lattice $P(\mathcal{S})$ of the fan is the lattice of cones ordered by inclusion and augmented with a maximal element 1. Thus, the coatoms (or facets) are the $d$-dimensional cones, the cocoatoms (or ridges) are their $(d-1)$-dimensional faces, the atoms (or vertices) are the one-dimensional cones (rays) and so on. A fan can alternatively be regarded as a spherical polytope, namely a realization of a complex on the unit sphere $S^{d-1}$, where all cells are spherical cells and their union covers $S^{d-1}$ (a spherical cell is the intersection $S^{d-1} \cap C$ of the sphere and a pointed polyhedral cone in $\mathbb{R}^{d}$ ).

Each convex polytope $K$ in $\mathbb{R}^{d}$ gives rise to a special fan, its normal fan, whose cones are in 1-1 correspondence with faces of $K$, where the cone corresponding to a face $G$ consists of those vectors $w \in \mathbb{R}^{d}$ for which the maximal value of the linear function $\langle w, \cdot\rangle$ over $K$ is attained at $G$. The face lattice of a convex polytope $K$ is isomorphic to the face lattice of the normal fan of its polar polytope $K^{*}$. However, not all fans arise that way, so face lattices of fans strictly include face lattices of convex polytopes. In contrast with the common belief that fans are not shellable in general [12], we have the following theorem.

Theorem 4.5. Face lattices of fans are signable.

Proof. Let $\mathcal{S}$ be a fan in $\mathbb{R}^{d}$. Pick a vector $g \in \mathbb{R}^{d}$ in general position with respect $\mathcal{S}$, so that $g$ does not lie in the linear $\operatorname{span} \operatorname{lin}(R)$ of any ridge $R$ of $\mathcal{S}$. We endow the face lattice $P=P(\mathcal{S})$ of $\mathcal{S}$ with a signing $\chi=\chi(\mathcal{S})$, thus turning it into a signed poset, as follows. Consider cones $R \prec F \prec 1$, and let $H$ be the hyperplane $\operatorname{lin}(R)$. All points in $F-R$ lie on one side of $H$. Put $\chi(R, F)=+$ if the vector $g$ and $F-R$ lie on the same side of $H$, and $\chi(R, F)=-$ if $H$ separates $g$ from $F-R$.

Let $\mathcal{P}$ be the family of signed face lattices of fans as above. We now show that $\mathcal{P}$ is an exact signed family: upper intervals correspond to orthogonal projections, and the unique positive cone in a fan $\mathcal{S}$ under the signing induced from $g$ is the one containing $g$.

Indeed, let $\mathcal{S}$ be a fan equipped with a vector $g$ as above, and let $P(\mathcal{S}) \in \mathcal{P}$ be the corresponding signed face lattice of $\mathcal{S}$. Let $[z, 1]$ be a strict signed upper interval in $P(\mathcal{S})$. In the space $\mathbb{R}^{d}$ where $\mathcal{S}$ is embedded, consider the linear span $Z$ of the face (cone) of $\mathcal{S}$ corresponding to $z$ and let $Z^{\perp}$ be its orthogonal complement. Project all faces (cones) of $\mathcal{S}$ in $[z, 1]$ on $Z^{\perp}$. This induces a fan $\mathcal{S}^{\prime}$ in $Z^{\perp}$ whose face lattice $P\left(\mathcal{S}^{\prime}\right)$ is isomorphic to the poset $[z, 1]$. Projecting $g$ on $Z^{\perp}$ as well, we obtain a vector $g^{\prime}$ which (after mapping $Z^{\perp}$ to $\mathbb{R}^{k}$ by an isometry) induces a signing of $P\left(\mathcal{S}^{\prime}\right)$ which makes it isomorphic to the signed interval $[z, 1]$. This simply follows from the fact that the property of being on the same side as $g$ of a hyperplane spanned by the vertices of a ridge $R$ in $\mathcal{S}$ and the origin is preserved for the images of ridges and for $g^{\prime}$ under the projection and the isometry. Thus, the family of signed face lattices of fans is a signed family.

Finally, it is easy to see that each such signed poset $P(\mathcal{S}) \in \mathcal{P}$ has a unique facet (maximal cone) $F \prec 1$ such that $\chi(R, F)$ is positive for all ridges $R \subset F$ : it is the unique cone of $\mathcal{S}$ containing the vector $g$. Hence $\mathcal{P}$ is an exact family. By Proposition 4.2 fans are signable. 
We conclude with the following result proved previously in [9]. The proof below is an easy consequence of our Theorems 4.4 and 4.5 . Here it is more convenient to use the realization of a fan $\mathcal{S}$ as a spherical polytope, so that its faces are the intersections $C \cap S^{d-1}$ of the various cones $C$ with the unit sphere rather than the cones themselves. In particular, the vertices (atoms) are points on $S^{d-1}$.

Corollary 4.6. Simplicial fans are partitionable and possess the upper-bound property.

Proof. The claim about partitionability follows from the previous theorem. We need to show that simplicial fans are totally signable. Let $\mathcal{S}$ be a fan and let $P=P(\mathcal{S})$ be its face lattice. Let $v$ be any vertex of $P$, which is a point $v \in S^{d-1}$. Choose a point $g$ on $S^{d-1}$ in general position with respect to $\mathcal{S}$, and sufficiently near to $v$, such that the hyperplane through $g$ and orthogonal to lin $(v)$ intersects only those facets of $S$ (intersections of $S^{d-1}$ with the full-dimensional cones) which contain $v$. Let $\chi_{v}$ be the resulting exact signing of $P(\mathcal{S})$. Consider any facet $F$ containing $v$. Then, by the choice of $g$, we have $\chi_{v}(F-v, F)=+$. Hence $v$ is positive in all facets containing it. Thus, simplicial fans are totally signable, and so, by Theorem 4.4 , possess the upper-bound property.

\section{Signability of Oriented Matroid Polytopes}

We now consider (so-called Las Vergnas) face lattices of oriented matroid polytopes. This is a large class which strictly contains the class of convex polytopes. It is believed that, in general, such face lattices are not shellable. In contrast, using the setup of the previous section, we prove that face lattices of oriented matroid polytopes are signable. In particular, we conclude that simplicial oriented matroid polytopes are partitionable and possess the upper bound property. We start with a quick review of some definitions and elementary properties of oriented matroids which will be needed in what follows. A comprehensive treatment of the subject can be found in [3].

An oriented matroid $M$ is a finite matroid having the following additional structure. Each circuit $C$ is endowed with a unique unordered partition $C=C^{+} \uplus C^{-}$such that the following holds: if $C_{1}$ and $C_{2}$ are two distinct circuits with partitions $C_{1}=C_{1}^{+} \uplus C_{1}^{-}$ and $C_{2}=C_{2}^{+} \uplus C_{2}^{-}$, and $v \in C_{1}^{+} \cap C_{2}^{-}$, then there is a circuit $C=C^{+} \uplus C^{-}$satisfying $C^{+} \subseteq C_{1}^{+} \cup C_{2}^{+}-v$ and $C^{-} \subseteq C_{1}^{-} \cup C_{2}^{-}-v$. The circuit $C$ is said to be obtained from $C_{1}$ and $C_{2}$ by eliminating $v$. It is known that, moreover, for any $u \in\left(C_{1}^{+}-C_{2}^{-}\right) \cup\left(C_{1}^{-}-C_{2}^{+}\right)$ there is a circuit $C$ as above that contains $u$.For each oriented matroid $M$ there is a unique oriented matroid $M^{*}$ on the same element set, called the dual of $M$, such that the following so-called orthogonality condition holds: a circuit $C=C^{+} \uplus C^{-}$of $M$ and a circuit $D=D^{+} \uplus D^{-}$of $M^{*}$ (a cocircuit of $M$ ) satisfy $\left(C^{+} \cap D^{+}\right) \cup\left(C^{-} \cap D^{-}\right) \neq \emptyset$ if and only if $\left(C^{+} \cap D^{-}\right) \cup\left(C^{-} \cap D^{+}\right) \neq \emptyset$. Naturally, $M^{* *}=M$, and the underlying matroids of $M$ and $M^{*}$ are dual to each other as (nonoriented) matroids as well. Note that, in particular, the orthogonality condition implies that, in the underlying matroids, if a circuit of $M$ and a circuit of $M^{*}$ (a cocircuit of $M$ ) intersect, then they have at least two elements in common. The deletion of $M$ by a subset $E$ of elements in $M$ is the oriented matroid $M-E$ whose circuits are the circuits in $M$ which do not intersect $E$. The cocircuits of $M-E$ are the minimal nonempty sets of the form $D-E$, where $D$ is a cocircuit of $M$. 
If $D=D^{+} \uplus D^{-}$is a cocircuit of $M$ and $D-E$ is a cocircuit of $M-E$ (which is always the case if $E \subseteq D)$, then its partition in $M-E$ is $D-E=\left(D^{+}-E\right) \uplus\left(D^{-}-E\right)$. The contraction of $M$ by $E$ is the oriented matroid $M / E=\left(M^{*}-E\right)^{*}$. The oriented matroid inherits the rank function $r$ of its underlying matroid. A flat in $M$ is a subset $G$ of $M$ such that $r(G+e)>r(G)$ for any element $e \notin G$ of $M$. The intersection of flats is again a flat. The corank of a subset $G$ of $M$ is $r(M)-r(G)$. A hyperplane is a flat of $M$ of corank 1 . Note that a subset of $M$ is a hyperplane if and only if its complement in $M$ is a cocircuit. A circuit $C$ of an oriented matroid is positive if its partition is $C=C^{+} \uplus \emptyset$. An oriented matroid is acyclic if it has no positive circuit. The deletion $M-E$ by any subset $E$ of elements of an acyclic $M$ is also acyclic.

A facet of an acyclic oriented matroid $M$ is the complement in $M$ of a positive cocircuit $D$ of $M$, and is in particular a hyperplane of $M$. A face of $M$ is any intersection of facets, and is in particular a flat. A ridge of $M$ is a face of corank 2 , and is the intersection of two distinct facets of $M$. The collection of faces of an acyclic oriented matroid $M$ is called the oriented matroid polytope of $M$, or the face lattice of the oriented matroid polytope (in [3] it is referred to as the Las Vergnas face lattice of $M$ ), and is denoted by $P(M)$. We regard $P(M)$ both as a collection of subsets of $M$ and as the poset of these sets ordered by inclusion and augmented with a maximal element 1 . Every oriented matroid polytope has a geometric realization as a piecewise linear sphere (see [3] for more on such topological representations). We adopt instead the point of view of a "generalized point configuration," motivated by the following example.

Example 5.1 (Point Configurations). Let $S$ be a finite set of points in real affine space $\mathbb{R}^{d}$. Then the collection of inclusion minimal affinely dependent subsets of $S$ is the collection of circuits of a matroid on $S$. Endowing each circuit with the unique Radon partition associated with its affine dependency, an acyclic oriented matroid $M$ is obtained: the affine oriented matroid of the point configuration $S$. It is known [10] that a subset $F$ of $S$ is a face of $M$ if and only if it is the intersection of $S$ with some hyperplane supporting $\operatorname{conv}(S)$ in $\mathbb{R}^{d}$. In particular, $P(M)$ equals the face lattice of $\operatorname{conv}(S)$.

We need the following two properties of acyclic oriented matroids. The proofs are provided in the Appendix.

Proposition 5.2. Let $M$ be an acyclic oriented matroid and let $G \in P(M)$ be a face of $M$. Then the contracted matroid $M / G$ is again an acyclic oriented matroid, and its face lattice is $P(M / G)=\{H-G: G \subseteq H \in P(M)\}$ which is the upper interval of $G$ in $P(M)$.

Proposition 5.3. Let $M$ be an acyclic oriented matroid, let $F$ be a facet of $M$, and let $G \subset F$ be a flat of $M$ of corank 2. Let $H$ be any hyperplane of $M$ satisfying $H \cap F=G$, and let $B=B^{+} \uplus B^{-}$be its complementing cocircuit. Then all elements of $F-G$ lie in the same part of $B$ if and only if $G$ is a ridge of $M$.

An element $e$ in a matroid $M$ is generic if $r(S+e)=r(S)+1$ whenever $S$ is a subset of $M$ of positive corank. If $G$ is any flat of $M$ of corank at least 2 and $e$ is a generic point, 
then $G+e$ is also a flat: for all $v \notin(G+e)$ we have

$r((G+e)+v)=r((G+v)+e)=r(G+v)+1=(r(G)+1)+1=r(G+e)+1$.

Also, if $C$ is a circuit containing a generic element $e$, then $C$ is necessarily of full rank. An element $e$ is an interior point in $M$ if it is not contained in any face of $M$ (equivalently, it is contained in all positive cocircuits of $M$ ). A rooted matroid is an acyclic oriented matroid $M$ with two distinguished elements $x, y$ which are both generic and interior in $M$. We endow the face lattice $P(M)$ of a rooted matroid $M$ with a signing $\chi=\chi(M)$, thus turning it into a signed poset, as follows. Let $x$ and $y$ be the distinguished elements of $M$ and consider a facet $F$ of $M$ and a ridge $R$ contained in it. Since $x, y$ are generic and interior we have that $x, y \notin R$ and $H=R+y$ is a hyperplane of $M$. Let $B=B^{+} \uplus B^{-}$ be the cocircuit complementing $H$ with $x \in B^{+}$. Define $\chi(R, F)=+$ if $F-R \subseteq B^{+}$ and $\chi(R, F)=-$ if $F-R \subseteq B^{-}$. Proposition 5.3 above guarantees that $\chi$ is well defined.

Let $\mathcal{P}$ be the family of signed face lattices of rooted matroids. We now prove a sequence of three lemmas which will establish that $\mathcal{P}$ is an exact signed family.

Lemma 5.4. The family $\mathcal{P}$ of signed face lattices of rooted matroids is a signed family.

Proof. Let $P(M) \in \mathcal{P}$ be any signed face lattice of a rooted matroid $M$ with distinguished elements $x, y$, and let $\chi(M)$ be its signing as above. Let $G$ be any face of $P(M)$, and let $L=M / G$ be the contracted oriented matroid. The elements $x, y$ are easily seen to be generic in $L$ as well; moreover, the cocircuits of $L$ are exactly those cocircuits $D$ of $M$ satisfying $D \cap G=\emptyset$, so all positive cocircuits of $L$ contain $x$ and $y$, which are therefore interior in $L$ as well. Thus, $L$ is again a rooted matroid with distinguished elements $x, y$, with signed face lattice $P(L) \in \mathcal{P}$. Let $\chi(L)$ be the signing of $P(L)$.

By Proposition 5.2, $P(L)=\{H-G: G \subseteq H \in P(M)\}$ is the upper interval $[G, 1]$ of $P(M)$. Let $F-G$ be any facet of $L$ and let $R-G$ be a ridge of $F-G$, where $F$ is a facet of $M$ containing $G$, and $R$ is a ridge of $F$ containing $G$. The complementing cocircuit $B$ of $(R-G)+y$ in $L$ is also the complement of $R+y$ in $M$. Also, $(F-G)-(R-G)=F-R$. Thus, $x$ and $F-R$ lie on the same part of the partition of $B$ in $M$ if and only if $x$ and $(F-G)-(R-G)$ lie on the same part of the partition of $B$ in $L$, which implies that $\chi(L)(R-G, F-G)=\chi(M)(R, F)$. Thus, $P(L)$ is the signed upper interval $[G, 1]$ of $P(M)$. Therefore the family $\mathcal{P}$ is closed under taking signed upper intervals, hence is a signed family.

We head on to prove that the family $\mathcal{P}$ above is exact. We need the following fundamental coloring theorem (Farkas' lemma type) of oriented matroids due to Lawrence and Bland [3, Theorem 10.2.14].

Proposition 5.5. Let $M$ be an oriented matroid and let $x, y$ be two distinct elements of $M$ such that $x$ is not a loop and $y$ is not a coloop. Then exactly one of the following alternatives holds:

1. There is a positive circuit $C=C^{+}$containing $x$ but not $y$, or a positive cocircuit $D=D^{+}$containing y but not $x$. 
2. A circuit $C=C^{+} \uplus C^{-}$and a cocircuit $D=D^{+} \uplus D^{-}$exist such that $y \in C^{+}$, $C^{-} \subseteq\{x\}, x \in D^{+}, D^{-} \subseteq\{y\}$, and $C \cap D \subseteq\{x, y\}$.

From this, we derive the following lemma.

Lemma 5.6. Let $M$ be an acyclic oriented matroid with a generic element $x$, and let $y$ be another element such that $x$ is contained in all positive cocircuits which contain $y$. Then a unique positive cocircuit $D$ in $M$ exists for which there is a circuit $C=C^{+} \uplus\{x\}$ satisfying $C \cap D=\{x, y\}$.

Proof. Since $M$ is acyclic, $x$ is not a loop. Since $x$ is in all positive cocircuits containing $y, y$ is not a coloop. Thus, the hypothesis of Proposition 5.5 holds, and the first alternative fails. Therefore the second alternative holds, so a circuit $C=C^{+} \uplus C^{-}$and a cocircuit $D=D^{+} \uplus D^{-}$exist such that $y \in C^{+}, C^{-} \subseteq\{x\}, x \in D^{+}, D^{-} \subseteq\{y\}$, and $C \cap D \subseteq$ $\{x, y\}$. Since $M$ is acyclic and $y \in C^{+}$, we must have $x \in C^{-}$, so $x \in C^{-} \cap D^{+}$. By orthogonality, then, since $C^{-} \cap D^{-}=\emptyset$, we must have $y \in C^{+} \cap D^{+}$. Thus, $D^{-}=\emptyset$ and $C \cap D=\{x, y\}$, so $C$ and $D$ satisfy the claim of the lemma.

Next, note that $C$ contains the generic element $x$, hence is of full rank $r(C)=r(M)=$ $r$. Assume, indirectly, that another positive cocircuit $B=B^{+}$, and a circuit $A=A^{+} \uplus\{x\}$ satisfying $A \cap B=\{x, y\}$, exist. Let $F$ be the complement of $D$ in $M$. Both $F$ and the complement of $B$ are flats of $\operatorname{rank} r-1$, so $F-B$ is a flat of corank at least 2 . Therefore it does not contain $C-\{x, y\}$, which is an independent set of rank $r-1$, so an element $v \in(C-\{x, y\}) \cap(F \cap B)$ exists. Thus, $v \in\left(C^{+} \cap B^{+}\right)-D^{+}$. Eliminating $x$ from the pair of cocircuits $B^{+} \uplus \emptyset$ and $\emptyset \uplus D^{+}$and keeping $v$, we find a cocircuit $S=S^{+} \uplus S^{-}$ such that $x \notin S, v \in S^{+} \subseteq B^{+}$, and $S^{-} \subseteq D^{+}$. Now, $v \in S^{+} \cap C^{+}$so by orthogonality, since $C^{-}=\{x\}$ and $x \notin S$, we must have $S^{-} \cap C^{+} \neq \emptyset$. However, $S^{-} \subseteq D^{+}$and $D^{+} \cap C^{+}=\{y\}$, so $y \in S^{-}$. Thus, $y \in S^{-} \cap A^{+}$, so by orthogonality of $A$ and $S$ we must have $S^{+} \cap A^{+} \neq \emptyset$. Nonetheless, $S^{+} \subseteq B^{+}$and $B^{+} \cap A^{+}=\{y\}$, yet $y \notin S^{+}$. Thus, $S^{+} \cap A^{+}=\emptyset$, which is the desired contradiction.

Lemma 5.7. The signed face lattice $P(M)$ of any rooted matroid $M$ contains a unique facet which is positive under its signing $\chi(M)$.

Proof. Let $x, y$ be the distinguished elements of $M$, and let $\chi=\chi(M)$. Let $F$ be any facet of $M$ and let $D$ be its complementing positive cocircuit. Since $x, y$ are generic, the circuits satisfying $C \cap D=\{x, y\}$ are exactly those sets $C=I \cup\{x, y\}$ with $I$ being a basis of $F$. Pick such a basis $I$ of $F$ and the corresponding circuit $C$, and write $C=C^{+} \uplus C^{-}$with $x \in C^{-}$. By orthogonality, $y \in C^{+}$.

Let $R$ be any ridge contained in $F$. Since $R$ is a flat of corank 2 , we have that $I-R \neq \emptyset$. Let $B$ be the cocircuit of $M$ complementing the hyperplane $R+y$, and write $B=B^{+} \uplus B^{-}$with $x \in B^{+}$, so that $x \in B^{+} \cap C^{-}$. Note that $B \cap C=(I-R)+x$. By Proposition 5.3 and the definition of $\chi$ following it, either $I-R \subseteq B^{+}$, in which case $\chi(R, F)=+$, or $I-R \subseteq B^{-}$in which case $\chi(R, F)=-$. In the second case, $(I-R) \cap C^{-} \neq \emptyset$ by orthogonality of $B$ and $C$, so $C^{-} \neq\{x\}$. Therefore, $C=C^{+} \uplus\{x\}$ only if $\chi(R, F)=+$ for all ridges $R \subset F$, i.e., if $F$ is a positive facet under $\chi$. Now, $M$ 
satisfies the hypothesis of Lemma 5.6 since $x$ is an interior point. Therefore a cocircuit $D=D^{+}$and a circuit $C=C^{+} \uplus\{x\}$ satisfying $C \cap D=\{x, y\}$ exist, and we conclude that $M$ has at least one positive facet under $\chi$.

We proceed to show that if $F$ is a positive facet, then its complementing cocircuit $D$ admits a circuit $C=C^{+} \uplus\{x\}$ satisfying $D \cap C=\{x, y\}$. The uniqueness of $F$ follows from the uniqueness part of Lemma 5.6. Let then $F$ be a positive facet with complement $D$, put $S=D-\{x, y\}$, and consider the deleted acyclic matroid $N=M-S$, whose element set is $F \cup\{x, y\}$. First, note that the points $x, y$ remain generic in $N$. Also, $\{x, y\}=D-S$ is a positive cocircuit in $N$, so $F$ remains a facet (and a hyperplane) in $N$. Next, consider any ridge $R$ of $F$ in $M$ and let $B=B^{+} \uplus B^{-}$be the cocircuit of $M$ complementing $R+y$, with $x \in B^{+}$. Then $S \subset B$, so $(F-R)+x=B-S=$ $\left(B^{+}-S\right) \uplus\left(B^{-}-S\right)$ is a cocircuit in $N$. Since $F$ is a positive facet, we have (Proposition 5.3 and the definition of $\chi)$ that $F-R \subset B^{+}$. Therefore $(F-R)+x$ is a positive cocircuit in $N$ (and its complement $R+y$ is a facet of $N$ ).

We now show that no hyperplane of $N$ containing $x$ but not $y$ is a facet of $N$. Let $H$ be such a hyperplane, and let $G=H \cap F=H-x$ be the corresponding flat of corank 2 . If $G$ is not a ridge of $N$, then, by Proposition 5.3, both parts of the cocircuit complementing $H$ in $N$ intersect $F$, so this cocircuit is not positive and $H$ is not a facet. Suppose then that $G$ is a ridge of $N$. Eliminating $x$ from the pair of positive cocircuits $((F-G)+x) \uplus \emptyset$ and $\emptyset \uplus\{x, y\}$, and keeping $y$, a cocircuit $B=B^{+} \uplus\{y\}$ is obtained, with $\emptyset \neq B^{+} \subseteq(F-G)$. However, then $B \subseteq(F-G)+y$ which is the cocircuit complementing $H$ in $N$, so that in fact this cocircuit equals $B$, which is not positive. Hence, once again, $H$ is not a facet of $N$. We conclude that $x$ belongs to all positive cocircuits of $N$ which contain $y$. Applying Lemma 5.6 to $N$, we find a circuit $C=C^{+} \uplus\{x\}$ containing $\{x, y\}$. However, then this is a circuit of $M$ as well, which intersects the complement $D$ of $F$ in $\{x, y\}$, as desired. This completes the proof.

Lemmas 5.7 and 5.4 finally yield the following result.

Theorem 5.8. The family $\mathcal{P}$ of signed face lattices of rooted matroids is an exact signed family.

We now show that any matroid polytope arises as the face lattice of some rooted matroid. For that, we need to discuss some material about extensions. An extension of an oriented matroid $M$ is an oriented matroid $M^{\prime}=M \cup E$, where $E$ is a new set of elements, such that the original matroid is the deletion $M=M^{\prime}-E$ of $M^{\prime}$. If $E=\{e\}$ consists of one element, then $M^{\prime}$ is a single element extension of $M$. If $M^{\prime}=M+e$ is an extension of $M$ and $D^{\prime}=D^{\prime+} \uplus D^{\prime-}$ is a cocircuit of $M^{\prime}$ with $e \in D^{\prime+}$, then $D^{\prime}-e=\left(D^{\prime+}-e\right) \uplus D^{\prime-}$ is a cocircuit of $M$. The following fundamental theorem on extensions due to Las Vergnas [3, Proposition 7.2.4] provides a certain converse of that fact.

Proposition 5.9. Let $\left(v_{1}, \ldots, v_{r}\right)$ be an ordered basis of $M$ and let $\left(\varepsilon_{1}, \ldots, \varepsilon_{r}\right) \in$ $\{-,+\}^{r}$ be an ordered tuple of signs. Then there is a unique single element extension $M\left(v_{1}^{\varepsilon_{1}}, \ldots, v_{r}^{\varepsilon_{r}}\right)=M^{\prime}=M+e$ of $M$ for which the following holds: if $D=D^{+} \uplus D^{-}$ is a cocircuit of $M$ and $v_{i} \in D^{\varepsilon_{i}}$, where $i$ is the smallest index such that $v_{i} \in D$, then $D+e=\left(D^{+}+e\right) \uplus D^{-}$is a cocircuit of $M^{\prime}$. 
The following proposition is an easy consequence of Proposition 5.9. We thank G. Ziegler for pointing it out.

Proposition 5.10. Let $M$ be an acyclic oriented matroid, let $\left(v_{1}, \ldots, v_{r}\right)$ be any ordered basis of $M$, and let $M^{\prime}=M\left(v_{1}^{+}, \ldots, v_{r}^{+}\right)=M+e$ be the corresponding single element extension. Then $M^{\prime}$ and $M$ have the same rank, $e$ is a generic and interior point in $M^{\prime}$, and $P\left(M^{\prime}\right)=P(M)$.

We can finally establish the main theorem of this section.

Theorem 5.11. Face lattices of oriented matroid polytopes are signable.

Proof. Let $M$ be an acyclic oriented matroid. Pick an ordered basis $\left(v_{1}, \ldots, v_{r}\right)$ of $M$ and let $M^{\prime}=M+y=M\left(v_{1}^{+}, \ldots, v_{r}^{+}\right)$. Then $\left(v_{1}, \ldots, v_{r}\right)$ is an ordered basis of $M^{\prime}$ as well. Let $M^{\prime \prime}=M^{\prime}+x=M^{\prime}\left(v_{1}^{+}, \ldots, v_{r}^{+}\right)$. Thus, $M^{\prime \prime}$ is obtained from $M$ by taking twice the single element extension with respect to $\left(v_{1}^{+}, \ldots, v_{r}^{+}\right)$. By Proposition 5.10, $M^{\prime \prime}$ and $M$ have the same oriented matroid polytope $P\left(M^{\prime \prime}\right)=P(M)$, and $x$ and $y$ are generic interior points in $M^{\prime \prime}$. Thus, $M^{\prime \prime}$ is a rooted matroid, and by Theorems 5.8 and 4.2 , its face lattice, which equals the face lattice of $M$, is signable.

Remark. If $F$ is any facet in $P(M),\left\{v_{1}, \ldots, v_{r-1}\right\}$ any basis of $F$, and $v_{r}$ any element outside of $F$, then taking the two-element extension $M^{\prime \prime}$ with respect to the ordered basis $\left(v_{1}, \ldots, v_{r}\right)$ as above, we find that $F$ itself will be the unique positive facet in the exact signing of $P(M)=P\left(M^{\prime \prime}\right)$ coming from the signed face lattice of the rooted matroid $M^{\prime \prime}$.

We end this section with the upper-bound property for simplicial oriented matroid polytopes.

Theorem 5.12. Simplicial oriented matroid polytopes are partitionable and possess the upper-bound property.

Proof. Let $M$ be a simplicial oriented matroid polytope, i.e., one whose face collection $P(M)$ is a simplicial complex $\Delta(M)$. The claim about partitionability follows from Theorem 5.11. We need to show that $\Delta(M)$ is totally signable. Let $v$ be any vertex of $\Delta(M)$. Endow $M$ with an ordered basis $\left(v_{1}, \ldots, v_{r}\right)$ in which $v=v_{1}$ is the first element, and let $M^{\prime}=M+y=M\left(v_{1}^{+}, \ldots, v_{r}^{+}\right)$and $M^{\prime \prime}=M^{\prime}+x=M^{\prime}\left(v_{1}^{+}, \ldots, v_{r}^{+}\right)$be the corresponding extensions. Then $M^{\prime \prime}$ is a rooted matroid which has the same simplicial oriented matroid polytope $\Delta\left(M^{\prime \prime}\right)=\Delta(M)$ as $M$. Let $\chi_{v}=\chi\left(M^{\prime \prime}\right)$ be the resulting exact signing of $\Delta(M)=\Delta\left(M^{\prime \prime}\right)$. Let $F$ be any facet containing $v$, and let $R=F-v$. Let $D^{\prime}$ be the cocircuit of $M^{\prime}$ which is the complement of the hyperplane spanned by $R+y$ in $M^{\prime}$, and write $D^{\prime}=D^{\prime+} \uplus D^{\prime-}$ with $v=v_{1} \in D^{\prime+}$. Then, by Proposition 5.9, $D^{\prime \prime}=D^{\prime}+x=\left(D^{\prime+}+x\right) \uplus D^{\prime-}$ is the cocircuit of $M^{\prime \prime}$ which is the complement of the hyperplane spanned by $R+y$ in $M^{\prime \prime}$. Therefore both $v$ and $x$ lie in $D^{\prime \prime+}$, and so $R$ is a positive ridge (and $v$ a positive vertex) of $F$ by definition of the signing $\chi_{v}$. Hence 
$v$ is positive in all facets containing it. Thus, simplicial oriented matroid polytopes are totally signable, and so by Theorem 4.4 possess the upper-bound property.

\section{Notes on Computational Complexity and Face Enumeration}

In this section we discuss the computational complexity of face enumeration and of deciding shellability and partitionability, and raise a few questions. Here all data is assumed to consist of rational numbers.

The following result of Linial [11] shows that, in general, enumeration of faces in a given complex, or elements in a poset, is computationally hard. We provide a short proof by direct reduction from Valiant's canonical $\# \mathcal{P}$-complete problem of computing the permanent of a $\{0,1\}$-valued matrix (the permanent of an $n$ by $n$ matrix $A$ is $\operatorname{perm}(A)=\sum_{\pi} \prod_{i=1}^{n} A_{i, \pi(i)}$, the sum extending over all permutations $\pi$ on $\left.[n]\right)$.

Proposition 6.1. Counting the number of vertices of a rational polytope $\{x: A x \leq b\}$ given as an intersection of half-spaces is \#P-complete.

Proof. The problem is easily seen to be in \#P. Let $A$ be a $\{0,1\}$-valued matrix of size $n$, the permanent of which is to be computed. Let

$$
P=\left\{X \in \mathbb{R}^{n \times n}: X_{i, j} \geq 0, \quad \sum_{i=1}^{n} X_{i, j}=\sum_{j=1}^{n} X_{i, j}=1,1 \leq i, j \leq n\right\}
$$

be the Birkhoff polytope of bistochastic $n$ by $n$ matrices. Since, as is well known, the vertices of $P$ are precisely the permutation matrices, it follows that

$$
\operatorname{perm}(A)=f_{1}(P \cap\{X:\langle A, X)=n\}),
$$

that is, the permanent of $A$ is given by the number of vertices of a suitable face of Birkhoff's polytope.

However, in the simplicial situation, things are better behaved, and signability can be used to compute face numbers, as we now demonstrate. Let $\mathcal{F}$ and $\mathcal{R}$ be finite sets representing facets and ridges, respectively, of a simplicial complex. Let

$$
\chi: \mathcal{R} \times \mathcal{F} \longrightarrow\{-, 0,+\}
$$

be an extended signing: $\chi(R, F) \neq 0$ means that the ridge $R \in \mathcal{R}$ is covered by the facet $F \in \mathcal{F}$, in which case $\chi$ gives the sign of $R$ in $F$. Note that $\mathcal{F}, \mathcal{R}$, and $\chi$ can be encoded in a number of bits proportional to the number $|\mathcal{R}| \cdot|\mathcal{F}|$ of ridge-facet pairs.

Lemma 6.2. There is a polynomial-time algorithm that, given $d \in \mathbb{N}$, finite sets $\mathcal{R}$ and $\mathcal{F}$, and an extended signing $\chi$ on $\mathcal{R} \times \mathcal{F}$, computes a vector $f=\left(f_{0}, \ldots, f_{d}\right)$ for which the following holds: if $\Delta$ is a simplicial complex of combinatorial dimension $d$ whose sets of facets and ridges could be put in bijections with $\mathcal{F}$ and $\mathcal{R}$, respectively, and which admits an exact signing which agrees with $\chi$ under these bijections, then $f$ is the $f$-vector of $\Delta$. 
Proof. From $\chi$, compute an $h$-vector $h=\left(h_{0}, \ldots, h_{d}\right)$ as described in Proposition 3.2, and apply the linear transformation to get an $f$-vector. If $\Delta$ satisfies the hypothesis of the lemma, then this computed $h$-vector, hence $f$-vector, equals those of $\Delta$.

In many situations a natural, say geometric, presentation of a simplicial complex can be used to produce, in polynomial time, sets $\mathcal{F}$ and $\mathcal{R}$ and exact signing $\chi$ as above, from which the face numbers can be computed via Lemma 6.2. As an example, we outline a proof of the following known fact, which stands in contrast with Proposition 6.1 above.

Proposition 6.3. It is possible to compute in polynomial time all face numbers of a simplicial convex polytope $K=\{x: A x \leq b\}$ given as an intersection of half-spaces.

Proof. It is possible, in polynomial time (using linear programming techniques), to check whether $K$ is nonempty, and if it is, transform it to an affinely equivalent polytope

$$
K=\left\{x: a_{i} x \leq b_{i}, i=1, \ldots, m\right\}
$$

of full affine dimension $d \geq 0$, such that $F_{i}=K \cap\left\{x: a_{i} x=b_{i}\right\}$ is a facet of $K$ for $i=1, \ldots, m$. The ridges are then determined as those intersections $R_{i, j}=F_{i} \cap F_{j}$ of affine dimension $d-2$. Thus, the sets $\mathcal{F}$ and $\mathcal{R}$ and the covering relations $R \prec F$ are determined. Next, a vector $g \in \mathbb{Q}^{d}$ can be computed that totally orders the facets by the value $\left\langle g, a_{i}\right\rangle$. This total order is a so-called line shelling of $K$. Therefore, by Theorem 3.6, the signing $\chi$ induced by this total order is exact. Lemma 6.2 now assures the efficient computability of the face numbers of $K$.

The signability of fans and oriented matroid polytopes also leads to efficient face enumeration in the simplicial case, under a suitable presentation of the object in question. We have the following result for fans, which extends the previous proposition. The analogous outcome for oriented matroid polytopes is omitted.

Theorem 6.4. It is possible to compute in polynomial time all face numbers of a simplicial fan $\mathcal{S}$ presented by the collection of its simplicial maximal cones $K_{i}=$ $\left\{x: A_{i} x \leq b_{i}\right\}, i=1, \ldots, m$.

Proof. The collection $\mathcal{F}$ of facets of $\mathcal{S}$ is simply $\mathcal{F}=\left\{K_{1}, \ldots, K_{m}\right\}$. The ridges are obtained as those intersections $R_{i, j}=K_{i} \cap K_{j}$ of affine dimension $d-1$. A vector $g \in \mathbb{Q}^{d}$ can be computed that does not lie in the linear span of any ridge, from which a signing $\chi$ is determined which by Theorem 4.5 is guaranteed to be exact. The face numbers of $\mathcal{S}$ are then obtained via Lemma 6.2.

We now turn to discuss the complexity of deciding shellability and partitionability. Here we assume a (pure) $d$-dimensional simplicial complex to have the vertex set $[m]=\{1, \ldots, m\}$ and to be presented as a list $F_{1}, \ldots, F_{n}$ of $d$-subsets of $[m]$ constituting its facets. Thus, the input size for computational complexity estimates can be taken as $n d$. 
Both shellability and partitionability are in the complexity class $\mathcal{N} \mathcal{P}$. Given a shellable simplicial complex, a shelling total order of its facets can be exhibited. Directly following the definition of a shelling in Section 2 , it is possible to verify efficiently that this is a shelling (namely in time polynomial in $n d$ ). Given a partitionable simplicial complex, an exact facing $\varphi$ can be exhibited. To verify that it is exact, it is necessary to check that each face $G \in \Delta$ is covered, namely satisfies $\varphi(F) \subseteq G \subseteq F$, by exactly one facet $F$ of $\Delta$. As shown by Noble [14], this can be efficiently done in the following way: checking that each face is covered by at most one facet can be done by verifying that $\varphi(F) \cup \varphi\left(F^{\prime}\right) \nsubseteq F \cap F^{\prime}$ for all pairs $F, F^{\prime}$ of facets; checking that each face is covered by at least one facet can be done by verifying, for each facet $F$, that the number of faces of $F$ covered by the various facets of $\Delta$ equals $2^{d}$.

We point out the following interesting fact and question. Consider the complexity of the following decision problem: given a simplicial complex $\Delta$ presented as above, and a positive integer $f$ presented in binary, does $f$ equal the total number of faces of $\Delta$ ? In other words, what is the complexity of deciding the set

$$
\mathcal{L}=\{(\Delta, f): f \text { is the total number of faces of } \Delta\} ?
$$

When restricted to the class of partitionable simplicial complexes, this decision problem is in $\mathcal{N P}$ : there is an exact signing $\chi$ which can be exhibited, from which the $f$-vector of $\Delta$, and hence the total number of faces, can be computed in polynomial time via Lemma 6.2 , and compared with the number $f$. What is the complexity of this decision problem for an arbitrary simplicial complex?

Next, we discuss the case of fixed dimension $d$. For our purposes, the input size in this case can be taken to be simply the number $n$ of facets. Clearly, both $d$-partitionability and $d$-shellability are in $\mathcal{N P}$ for any $d$. For $d \leq 2$ both are decidable in polynomial time, the simplicial complexes in question being simply graphs. For any $d \geq 4$ the polynomial-time decidability is open. In dimension 3 (geometric dimension 2), if attention is restricted to pseudomanifolds, i.e., connected simplicial complexes in which every ridge is contained in either one or two facets, then the following result of Danaraj and Klee [5] is available.

Proposition 6.5. Shellability of three-dimensional pseudomanifolds is polynomialtime decidable.

The proof is based on the interesting fact, proved in [5], that any shellable threedimensional pseudomanifold is in fact extendibly shellable, i.e., any partial shelling of it extends to a shelling. Thus, the following polynomial-time greedy-type algorithm decides shellability of such complexes: pick up facets of the given $\Delta$ in a greedy fashion, maintaining the property that the facets $F_{1}, \ldots, F_{k}$ picked so far form a partial shelling. The next facet $F_{k+1}$ can be taken to be any facet $F$ not picked yet, which extends the partial shelling, i.e., satisfies the property that for all $1 \leq i \leq k$ there is an $r \leq k$ such that $F_{i} \cap F \subseteq F_{r} \cap F$ and $F_{r} \cap F$ is a ridge of $\Delta$. The three-dimensional complex is shellable if and only if this algorithms terminates with a shelling. Note that in higher dimensions this algorithm may fail. 
We conjecture that, in contrast, deciding whether a three-dimensional pseudomanifold is partitionable is already $\mathcal{N} \mathcal{P}$-complete. This would follow if a switching simplicial complex, which we now define, exists. We say that a facing $\varphi$ of a simplicial complex $\Delta$ is a $\Sigma$-facing, where $\Sigma$ is a subcomplex of $\Delta$, if the following hold: every face in $\Delta-\Sigma$ is covered by exactly one facet of $\Delta$ under $\varphi$, whereas every face in $\Sigma$ is covered by no face of $\Delta$ under $\varphi$. For example, a $\Sigma$-facing is an exact facing of $\Delta$ if and only if $\Sigma=\emptyset$ is the empty complex. We call $\Delta$ a switching simplicial complex if it has three distinguished vertices $v_{1}, v_{2}, v_{3}$ for which the following hold:

1. $\Gamma=\left\{\left\{v_{1}\right\},\left\{v_{2}\right\},\left\{v_{3}\right\}, \emptyset\right\}$ is the collection of all faces of $\Delta$ which are contained in $\left\{v_{1}, v_{2}, v_{3}\right\}$.

2. $\Delta$ admits a $\Sigma$-facing with a nonempty $\Sigma \subseteq \Gamma$ if and only if $\Sigma=\Gamma$ or $\Sigma=\{\emptyset\}$.

Proposition 6.6. For any $d \geq 3$, if a $d$-dimensional switching simplicial complex exists, then $d$-partitionability is $\mathcal{N} \mathcal{P}$-complete .

Proof. The following problem is known to be $\mathcal{N} \mathcal{P}$-complete [7]: given a collection $\left\{S_{1}, \ldots, S_{n}\right\}$ of 3 -subsets of $[m]=\{1, \ldots, m\}$, decide if there is a subcollection forming a partition of $[m]$, i.e., a subcollection $\left\{S_{i}: i \in I\right\}$ whose members are pairwise disjoint and their union equals $[m]$. If a $d$-dimensional switching complex $\Delta$ exists, then it can be used as a building block to reduce this problem to $d$-partitionability in polynomial time. The details are omitted.

It would be interesting to settle the question of whether or not there is any $d$ for which a $d$-dimensional switching simplicial complex exists.

As a final remark about the algorithmic aspects of shellability and partitionability, we mention the remarkable fact that starting in dimension 6 (geometric dimension 5) it is undecidable whether or not a given simplicial complex $\Delta$ is a sphere [19]; but if $\Delta$ is a pseudomanifold in which each ridge lies in exactly two facets and is shellable, then it is a sphere. Note that in contrast, there are nonspherical partitionable pseudomanifolds in which each ridge lies in exactly two facets, such as the real projective plane of Example 2.1. See [6] for more details.

\section{Final Remarks}

In view of the various classes of posets and simplicial complexes studied in this paper, it would be interesting to settle several open questions concerning the hierarchy suggested by Fig. 1. For example, it is known that the Barnette sphere (see [3]) is a fan but not an oriented matroid polytope; can an oriented matroid polytope which is not a fan be found? Also, as raised by J. Bokowski, can a fan be constructed which is also an oriented matroid polytope, but is not convex?

As mentioned earlier, it is believed that fans and oriented matroid polytopes are nonshellable in general; but the resolution of either seems difficult at present. It is also open, and of great interest, whether all spheres are signable (see Fig. 1). 


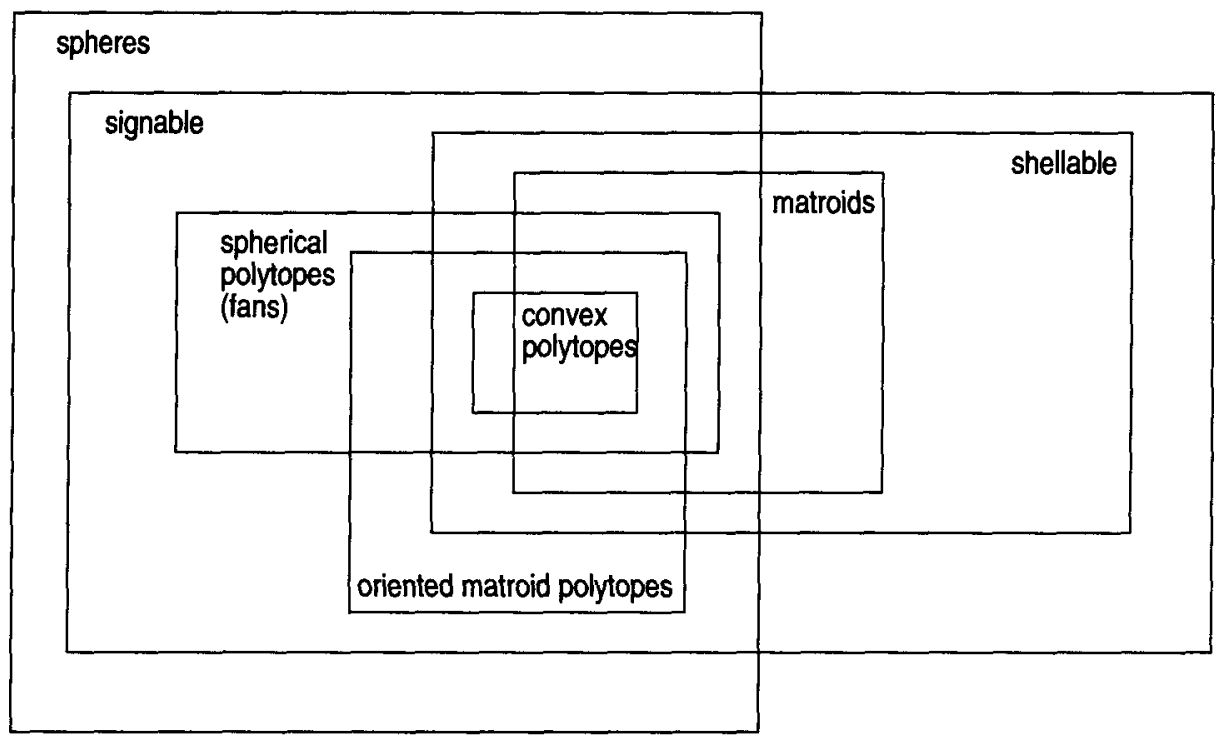

Fig. 1. A hierarchy of posets and simplicial complexes.

Another topic of interest circles around face rings of partitionable complexes. With each $d$-dimensional simplicial complex with $n$ vertices is associated its so called StanleyReisner ring, a quotient of a polynomial ring $\mathcal{K}[X]$ (where $\mathcal{K}$ is a field and $X=$ $\left(x_{1}, \ldots, x_{n}\right)$; see [17] for details). When this ring is Cohen-Macaulay, then, factoring out a system of parameters, a finite dimensional graded $\mathcal{K}$-algebra $\mathcal{A}=\bigoplus_{i=0}^{d} \mathcal{A}_{i}$ is obtained, and the dimensions $\operatorname{dim}_{\mathcal{K}}\left(\mathcal{A}_{i}\right)=h_{i}$ form the $h$-vector of $\Delta$. If $\varphi$ is an exact facing coming from a shelling of $\Delta$ (see Section 2), then the (residue classes of the) monomials $X^{\varphi(F)}$ form a $\mathcal{K}$-space basis for $\mathcal{A}[8]$. The role of these monomials when $\varphi$ is an exact facing not coming from a shelling is not clear and should be the subject of future study. An interesting open question here is whether all Cohen-Macaulay complexes are partitionable.

Concluding, in this article we have introduced the class of signable posets, and shown that it includes shellable posets and face lattices of partitionable complexes, fans, and oriented matroid polytopes. We have used signability in proving the upper-bound theorem and for efficient face enumeration for various complexes in these classes. In a continuation article [15], poset signings are further investigated, and are shown to play an important role in the algorithmic and enumerative study of recursively signable posets, their flag vectors, and their chain complexes and barycentric subdivisions.

\section{Acknowledgments}

The second author is indebted to Bernd Sturmfels for introducing him to the theory of oriented matroids. He thanks Jürgen Bokowski for remarks regarding the hierarchy in Fig. 1, Günter Ziegler for very helpful conversations and suggestions, and the referees for their comments. He also thanks Universität Passau for its hospitality and the Alexander von Humboldt Stiftung for its support while a major part of this work was done. 


\section{Appendix}

Here we provide proofs omitted from the main text.

Proposition 5.2. Let $M$ be an acyclic oriented matroid and let $G \in P(M)$ be a face of $M$. Then the contracted matroid $M / G$ is again an acyclic oriented matroid, and its face lattice is $P(M / G)=\{H-G: G \subseteq H \in P(M)\}$ which is the upper interval of $G$ in $P(M)$.

Proof. Suppose indirectly that $C^{\prime}=C^{\prime+}$ is a positive circuit in $M / G$. Then there is a circuit $C=C^{+} \uplus C^{-}$in $M$ such that $C^{\prime+}=C^{+}-G$ and $C^{-}=C^{-}-G$, so that $C^{+}-G \neq \emptyset$ and $\emptyset \neq C^{-} \subseteq G$. If now $\bigcup D_{i}$ is a union of positive cocircuits which is the complement of $G$ in $M$, we find that $C^{+} \cap D_{i} \neq \emptyset$ for some $D_{i}$, whereas $C^{-} \cap D_{i}=\emptyset$, contradicting the orthogonality property for $C$ and $D_{i}$. Thus, $M / G$ is acyclic.

Next, note that the cocircuits of $M / G$ are exactly those cocircuits $D$ of $M$ satisfying $D \cap G=\emptyset$. Thus, $F$ is a facet of $P(M / G)$ if and only if $F \cup G$ is a facet in $M$. The rest of the claims follow since each face is an intersection of facets.

Proposition 5.3. Let $M$ be an acyclic oriented matroid, let $F$ be a facet of $M$, and let $G \subset F$ be a flat of $M$ of corank 2. Let $H$ be any hyperplane of $M$ satisfying $H \cap F=G$, and let $B=B^{+} \uplus B^{-}$be its complementing cocircuit. Then all elements of $F-G$ lie on the same part of $B$ if and only if $G$ is a ridge of $M$.

Proof. We claim that the following holds for any oriented matroid: let $F$ be any hyperplane and $G \subset F$ a flat of corank 2. Then $F-G$ is partitioned in the same way by all cocircuits complementing hyperplanes meeting $F$ in $G$. This claim will establish the "if" direction: if $F$ is a facet and $G$ is a ridge, then there is another facet $F^{\prime}$ such that $G=F \cap F^{\prime}$; the complement of $F^{\prime}$ is a positive cocircuit, so contains all elements of $F-G$ on the same part. Therefore all elements of $F-G$ lie on the same part of any cocircuit complementing a hyperplane $H$ satisfying $H \cap F=G$. To prove the claim, suppose indirectly that there are two hyperplanes $H_{1}, H_{2}$ violating it. Thus, $H_{1} \cap F=H_{2} \cap F=G$ and, letting $B_{i}=B_{i}^{+} \uplus B_{i}^{-}$be the cocircuit complementing $H_{i}(i=1,2)$, we may assume that there are two elements $u, v \in F-G$ satisfying $u \in B_{1}^{+} \cap B_{2}^{+}$and $v \in B_{1}^{+} \cap B_{2}^{-}$. Let $B=B^{+} \uplus B^{-}$be the cocircuit obtained from $B_{1}$ and $B_{2}$ by eliminating $v$ and keeping $u$, and let $H$ be the complement of $B$. Since $B \subset B_{1} \cup B_{2}$ and $G \cap\left(B_{1} \cup B_{2}\right)=\emptyset$, we have $G \subset H$. Now, $v \notin B$ so $v \in H$ and, since $v \in F-G$, we must have $H=F$. However, $u \in F \cap B=F-H$ which is a contradiction. The claim and the "if" direction are proved.

Suppose now that $G$ is not a ridge and that, indirectly, there is a hyperplane meeting $F$ in $G$, whose complement is a cocircuit with $B^{+} \cap F \neq \emptyset$ and $B^{-} \cap F=\emptyset$. Pick such a hyperplane with $\left|B^{-}\right|$as small as possible. This hyperplane is not a facet since $G$ is not a ridge, hence $B^{-}$is nonempty. Let $D=D^{+}$be the complement of $F$, and pick an element $v \in B^{-} \subset D$. Also, pick an element $u \in B-D$ (so $u \in B^{+}$). Let $A=A^{+} \uplus A^{-}$ be a cocircuit obtained by eliminating $v$ from the pair $D^{+} \uplus \emptyset$ and $B^{+} \uplus B^{-}$and keeping $u$. Then $A^{-} \subset B^{-}-v$ and $u \in A^{+} \subset D^{+} \cup B^{+}$. Now

$$
A \cap F=A^{+} \cap F \subseteq B^{+} \cap F=B \cap F,
$$


and $G$ is the intersection of $F$ with the complement of $B$, so letting $H$ be the hyperplane complementing $A$, we find that $G \subseteq H \cap F$. Since $G$ is a flat, this implies that either $G=H \cap F$ or $H=F$. However, the last possibility is impossible, since $u \in A \cap F$. Hence $H \cap F=G$, and $\left|A^{-}\right|<\left|B^{-}\right|$, which contradicts the choice of $B$ and its complementing hyperplane.

\section{References}

1. L. J. Billera and J. S. Provan. Decomposition of simplicial complexes related to diameters of convex polyhedra. Math. Oper. Res., 5:576-594, 1980.

2. A. Björner. Homology and shellability of matroids and geometric lattices. In N. White, editor, Matroid Applications, chapter 7, pages 226-283. Cambridge University Press, Cambridge, 1992.

3. A. Björner, M. Las Vergnas, B. Sturmfels, N. White, and G. M. Ziegler. Oriented Matroids. Cambridge University Press, Cambridge, 1993.

4. A. Björner and M. Wachs. On lexicographically shellable posets. Trans. Amer. Math. Soc., 277:323-341, 1983.

5. G. Danaraj and V. Klee. A representation of 2-dimensional pseudomanifolds and its use in the design of a linear-time shelling algorithm. Ann. Discrete Math., 2:53-63, 1977.

6. G. Danaraj and V. Klee. Which spheres are shellable? Annals of Discrete Mathematics, 2:33-52, 1978.

7. M. R. Garey and D. S. Johnson. Computers and Intractability. Freeman, San Francisco, CA, 1979.

8. B. Kind and P. Kleinschmidt. Schälbare Cohen-Macaulay Komplexe und ihre Parametrisierung. Math. Z., 167:173-179, 1979.

9. P. Kleinschmidt and Z. Smilansky. New results for simplicial spherical polytopes. In J. E. Goodman, R. Pollack, and W. Steiger, editors, Special Year on Discrete and Computational Geometry, pages 187 197. DIMACS Series, vol. 6. American Mathematical Society, Providence, RI, 1991.

10. M. Las Vergnas. Convexity in oriented matroids. J. Combin. Theory Ser, B, 29:231-243, 1980.

11. N. Linial. Hard enumeration problems in geometry and combinatorics. SIAM J. Algebraic Discrete Methods, 7:331-335, 1986.

12. P. Mani-Levitska. Convex polytopes and smooth structures on manifolds. Euroconference on Combinatorial Geometry, Crete, July 1994.

13. P. McMullen. The maximum numbers of faces of a convex polytope. Mathematika, 17:179-184, 1970.

14. S. D. Noble. Recognizing a partitionable simplicial complex is in $\mathcal{N P}$. Discr. Math., to appear.

15. S. Onn. Partitionable Posets. Manuscript, 20 pages, 1995.

16. R. Seidel. Constructing higher dimensional convex hulls at logarithmic cost per face. Proc. 18th Ann. ACM Symp. on Theory of Computing, pages 404-413, 1986.

17. R. P. Stanley. Combinatorics and Commutative Algebra. Birkhäuser, Boston, 1983.

18. R. P. Stanley. Enumerative Combinatorics, vol. 1. Wadsworth and Brooks/Cole, Pacific Grove, CA, 1986.

19. I. A. Volodin, V. E. Kuznetsov, and A. T. Fomenko. The problem of discriminating algorithmically the standard three-dimensional sphere. Russian Math. Surveys, 29:71-172, 1974.

Received September 8, 1994, and in revised form September 14, 1995, and November 16, 1995. 